\title{
Study of the effectiveness of various cannabinoid receptor 1 (CB1) agonists using molecular docking and molecular dynamics modeling
}

Volodymyr V. Tkachenko a*, Volodymyr S. Farafonov", Viktor V. Tokarev ${ }^{\mathrm{a}}$, Irina G. Tkachenko ${ }^{\mathrm{a}, \mathrm{b}}$

${ }^{a}$ School of Chemistry, V. N. Karazin Kharkiv National University, Svobody Sq., 4, Kharkiv 61022, Ukraine

${ }^{\mathrm{b}}$ Kharkiv Scientific Research Forensic Center, Ministry of Internal Affairs of Ukraine, Kovtuna str., 32, Kharkiv 61036, Ukraine

\section{v.v.tkachenko@karazin.ua}

Keywords: cannabinoid receptor 1, molecular docking, molecular dynamics simulations, agonists, binding energy.

The binding of a series of small organic molecules, acting as agonists of the cannabinoid receptor $\mathrm{CB} 1$, was investigated by means of three methods of computational chemistry. Binding modes were predicted by means of molecular docking, and binding free energy was estimated via docking, molecular-mechanics Poisson-Boltzmann surface area method, and multistate Bennett acceptance ratio. No evident correlation was observed for the molecules between the experimental characteristics of affinity and three computed binding free energy estimates. The reasons for the discrepancy were discussed.

\section{Introduction}

The endocannabinoid system (ECS) is a perspective therapeutic target, which participates in multiple physiological processes, such as appetite stimulation, immune response, vomiting control, pain modulation [1-3], and also in pathological conditions, for example Huntington's disease and stroke [4, 5].

$\mathrm{CB} 1$ and $\mathrm{CB} 2$ receptors constitute most recognized part of ECS. Their natural agonists, so-called endocannabinoids, are anandamide and 2-arachidonoylglyerol. Another well-known partial agonist is $\Delta^{9}$-tetrahydrocannabinol (THC), the natural component of plant
Cannabis sativa, which is used for centuries for its recreational and medicinal properties $[1,6]$.

In last decades new potent agonists of CB1 and CB2 receptors were synthesized. While initially they were developed with legitimate research purposes, synthetic cannabinoids started new era of "designer drugs", semi-legal replacements of regulated psychoactive compounds. While most of these synthetic cannabinoids are already known to scientific community, their structural diversity presents unlimited possibilities for further modification, thus evading state regulation $[7,8]$. 
Fast development of synthetic cannabinoids poses significant treat for public health. They have low binding affinity and are not subject to any clinical tests, so overdosing is frequent, with typical symptoms including anxiety and panic attacks $[9,10]$.

Earlier studies had to use QSAR to predict affinity of synthetic cannabinoids [11]. But recent publication of high precision CB1 $[12,13]$ and CB2 [14] crystal structures allows to estimate and explain binding affinity using more reliable methods of molecular docking [15] and molecular dynamics [16,17].

We expect that comparison of computed binding affinity of known agonists will allow to develop high-quality agonist pharmacophore models. This, along with purely pharmacological applications, will make robust identification and classification of narcotic drugs with THC-like action, and pave the path for estimation of their health risks.

\section{Experimental part}

\section{Computational Methods}

We chose three principally different approaches to estimate binding free energies of ligands to CB1. The first one is the common method of molecular docking, and the others are based on molecular dynamics (MD) simulation. The second one is Molecular Mechanics Poisson-Boltzmann Surface Area (MMPBSA) [18] approach. It is but approximate but computationally cheap and parameterized specifically for protein-ligand systems. The third method is multistate Bennett acceptance ratio (MBAR) from the family of alchemical transformation approaches $[19,20]$. It is a rigorous method of universal applicability but is costly because requires longer equilibration and several additional simulations to be performed.

Protein preparation for docking. The initial structure of CB1 protein in active apoconformation was taken from the paper [17]. There, it was reconstructed from the crystal structure available in Protein Data Bank (ID: 5XRA [12]). Namely, the missing and mutated parts were restored, and equilibration via molecular dynamics in physiological conditions (immersed into lipid bilayer solvated in $0.15 \mathrm{M}$ $\mathrm{NaCl}$ solution) was performed.

The final structure of the intact active conformation of $\mathrm{CB} 1$ protein for docking was prepared using the Structure Preparation module of the Molecular Operating Environment (MOE) software [21]. The protonation and tautomeric states of Arg, Asp, Glu, His and Lys residues were adjusted to match $\mathrm{pH}=7.0$ using the Protonate 3D module. Restrained minimization with the AMBER forcefield was performed via the Energy Minimize panel.

Ligand preparation for docking. Wellknown cannabinoid receptor CB1 agonists were selected for our study (Figure 1): widely studied THC, three $N$-pentylindole derivatives (JWH-018， UR-144， PB-22) and their monofluorinated derivatives (AM-2201, XLR11, 5F-PB-22, respectively). All these ligands 
have $\mathrm{K}_{\mathrm{i}}$ values $<50 \mathrm{nM}$ according to the initially drawn using the ChemDraw

ChEMBL database.

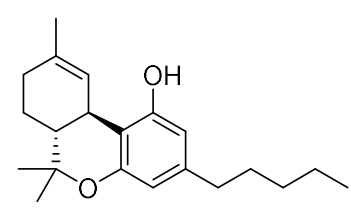

Tetrahydrocannabinol (THC)

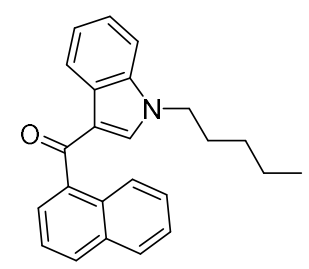

JWH-018

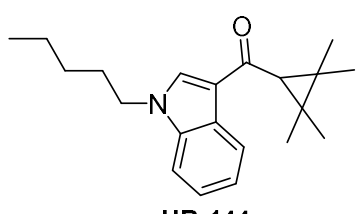

UR-144

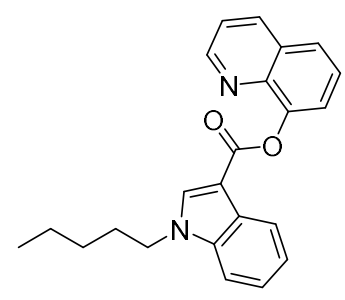

PB-22

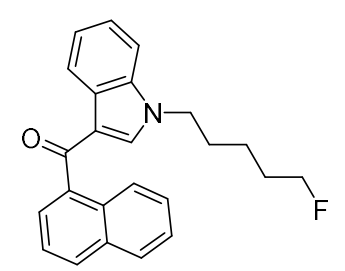

AM-2201

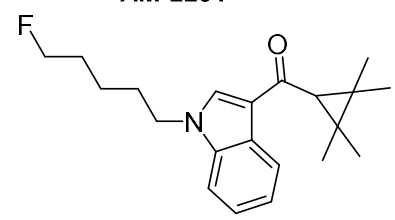

XLR-11

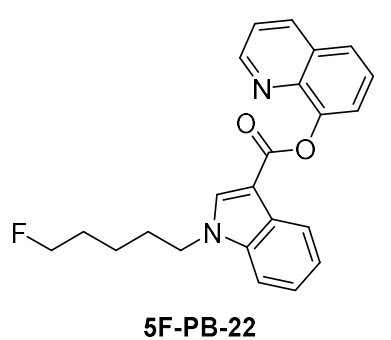

5F-PB-22

Figure 1. Agonist structures selected for investigation.

All ligand structures (Figure 1) for $\Delta^{9}-$ tetrahydrocannabinol (THC), naphthalen-1-yl(1pentyl-1H-indol-3-yl)methanone (JWH-018), (1-(5-fluoropentyl)-1H-indol-3-yl)(naphthalen1-yl)methanone (AM-2201), (1-pentyl-1Hindol-3-yl)(2,2,3,3-

tetramethylcyclopropyl)methanone

(UR-144),

(1-(5-fluoropentyl)-1H-indol-3-yl)(2,2,3,3-

tetramethylcyclopropyl)methanone (XLR-11), quinolin-8-yl-1-pentyl-1H-indole-3-carboxylate (PB-22) and quinolin-8-yl 1-(5-fluoropentyl)1H-indole-3-carboxylate (5F-PB-22) were

Professional and prepared using the LigX module of MOE. LigX generated stereoisomers and tautomers within a $\mathrm{pH}$ range of $7.0 \pm 2.0$. For each ligand, the lowest energy conformer was retained. Next, the ligands were optimized via energy minimization using the "AMBER10:EHT" forcefield while allowing adjustment to $\mathrm{H}$ atoms. The RMSD gradient value was kept at $0.1 \mathrm{kcal} / \mathrm{mol} \cdot \mathrm{A}^{2}$ (energy minimization is terminated when RMSD gradient falls below this value). The calculated electrostatic potential charges were used as partial charges of ligand atoms.

Molecular docking. The ligands were docked to the orthosteric binding site of the active conformation of the CB1 structure by using the Dock panel of MOE software. We decided to dock the selected ligands only for active conformation of the $\mathrm{CB} 1$ receptor, since, according to Jung et al [17], agonists more favorably interacted with the active form and had lower binding energies to the inactive one.

The induced-fit docking (IFD) protocol was used in all cases. The receptor was kept at "Receptor Atoms" while receptor site was chosen to be "Selected atoms". No "wall constraints" were used. All docking jobs were performed with the placement method "Triangle Matcher" with post placement refinement kept as "Forcefield". The initial scoring function was kept as "London dG", and "GBVI/WSA dG" was used as the final scoring function [22]. The 
pose with the lowest docking score (S-score) was retained for each ligand.

\section{MD simulations setup}

The initial structures of $\mathrm{CB} 1$ protein in active apo- conformation and in active conformation with bound THC were taken from the original paper [17]. They consist of the protein embedded to a 1-palmitoyl-2oleoylphophatidylcholine (POPC) bilayer surrounded by $0.15 \mathrm{M} \mathrm{NaCl}$ solution, and are equilibrated via MD run without (apoconformation) or with THC inside.

Here, as initial configurations for MD, the same structure was employed with a ligand placed inside the protein in a pose predicted by molecular docking (Figure 2). At the same time, four water molecules initially found inside the empty apo- protein were removed. In the case of THC, we used the original structure from [17] without modifications.

The distribution of protonation states in the initial structure was preserved; it matches $\mathrm{pH}=$ 7.0. Particularly, His residues are neutral, with $\mathrm{H}$ atom bonded to $\mathrm{N}^{\delta}$ (acronym "HSD").

We employed two forcefields for the computations. Firstly, following the original study [17], CHARMM36 and CGenFF forcefields [23,24] were used in order to keep compatibility. Potential model for POPC is already present in it, while the ones for ligands were prepared using ParamChem web server. As usual for CHARMM, water was described with TIP3P model.

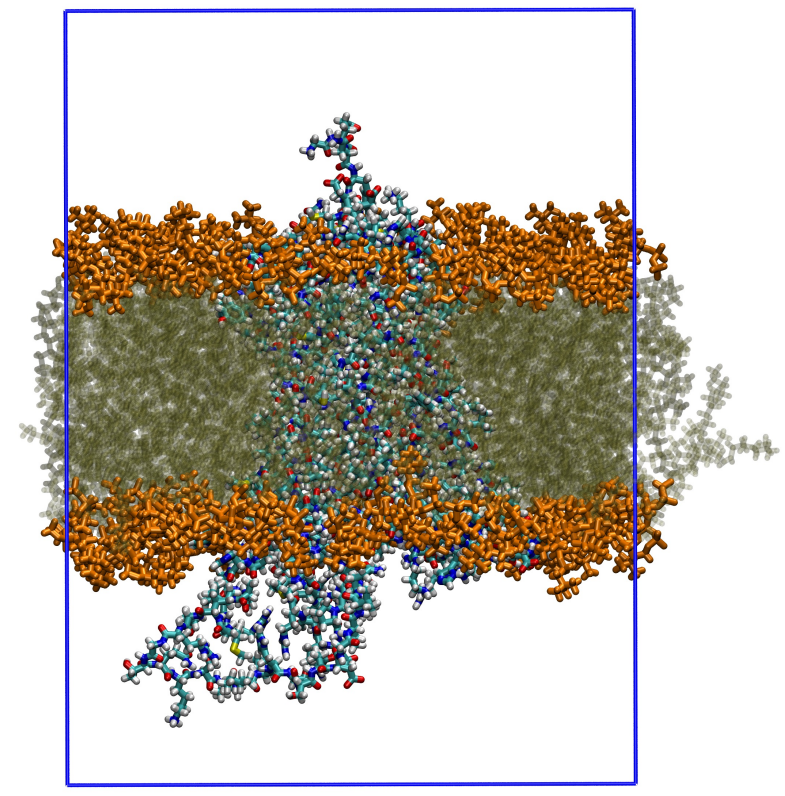

Figure 2. Initial configuration for MD simulations with CB1 in apo- conformation. Phophatidylcholine groups are colored orange, while the rest of POPC molecules is colored brown. Water, $\mathrm{NaCl}$, and ligand are not shown.

The second one was OPLS-AA/M forcefield [25] that is a recent update of a wellknown and validated OPLS-AA forcefield, containing improved parameters for peptide dihedral angles. For POPC, a compatible modern potential model was taken from [26, 27]. It is developed in the framework of OPLSAA, but contains some changes in parameters describing dihedral angles allowing it to reproduce the properties of POPC bilayers well. For ligands, the potential models were prepared with the help of LigParGen server, which is tailored for the OPLS-AA/M forcefield [28]. Because all the ligands were neutral molecules, the atomic point charges were computed using $1.14 *$ CM1A model with LBCC correction. 
Finally, water model was TIP3P because POPC was parameterized together with it [27].

Molecular dynamics simulations was carried out using GROMACS 5 software. For CHARMM36 runs, we reproduced the settings from [17]. Namely, temperature $310 \mathrm{~K}$ and pressure 1 bar were maintained by means of Nose-Hoover thermostat and Parrinello-Rahman barostat with semiisotropic coupling, respectively; time constants equaled 1 ps. The parameters were as follows: time step 2 fs, 3D periodic boundary conditions, constraints on all bonds involving hydrogen atoms, PME method for computing electrostatics with real space cutoff $1.2 \mathrm{~nm}$, cutoff of van der Waals interactions at $1.2 \mathrm{~nm}$ with force-switch at $1 \mathrm{~nm}$. The duration of a run was $5 \mathrm{~ns}$ for each ligand.

For OPLS-AA/M runs, some settings were changed: thermostat time constant was 0.4 ps, constraints on all bonds, PME real space cutoff $1 \mathrm{~nm}$, plain cutoff of van der Waals interactions at $1 \mathrm{~nm}$. The same and settings were employed during parameterizing POPC [27]. Simulations ran for $10 \mathrm{~ns}$ because additional equilibration of the initial configurations (originally made in CHARMM36 forcefield) was needed.

The MMPBSA computations were performed using g_mmpbsa program [29,30]. Polar interactions were treated by non-linearized version of Poisson-Boltzmann equation for higher accuracy, apolar ones were calculated basing on solvent-accessible surface areas, the rest of settings were set at default values (in particular, eco $=80$, pdie $=2, \operatorname{srad}=1.4$ ). For processing, protein-ligand complex configurations were extracted each 20 ps from the last 5 ns (OPLS-AA/M) or 3 ns (CHARMM36) of MD trajectories.

Because MBAR method requires simulating a single system at several values of coupling parameter $\lambda$, two series of additional simulations were carried out for each ligand. Firstly, each ligand was simulated in CB1 at each $\lambda$ value for $6 \mathrm{~ns}$ starting from the final configuration of its ordinary MD run. The first 3 ns were discarded as equilibration and were not used for calculations. Secondly, each ligand was solvated in a water box with size $6.5 \mathrm{~nm}$ and then simulated at each $\lambda$ value for $3 \mathrm{~ns}$; the first 1 ns was omitted. The following $\lambda$ schedules were used: $\{0.0 ; 0.2 ; 0.4 ; 0.6 ; 0.8 ; 1.0 ; 1.0\}$ for electrostatic interactions and $\{0.0 ; 0.2 ; 0.4$; $0.6 ; 0.8 ; 0.9 ; \quad 1.0\}$ for van der Waals interactions, where $\lambda$ values 0.0 and 1.0 correspond to the specified interactions between ligand and environment turned on and off, respectively. The difference between schedules ensures the electrostatic interactions are disabled before the van der Waals ones are that is required to avoid singularities. For the last $3 \lambda$ values, stochastic dynamics integrator was used, as is recommended for proper sampling.

\section{Results and discussion}

Docking results 
All agonist ligands studied were docked to the CB1 active conformation by molecular docking to predict the ligand binding pose at the orthosteric binding site. The docking results for some agonists, namely XLR-11, PB-22 and 5FPB-22 are shown in Figures 3-5, respectively. All ligands were well fixed in the active conformation of the receptor and showed similar binding poses at the orthosteric binding site.

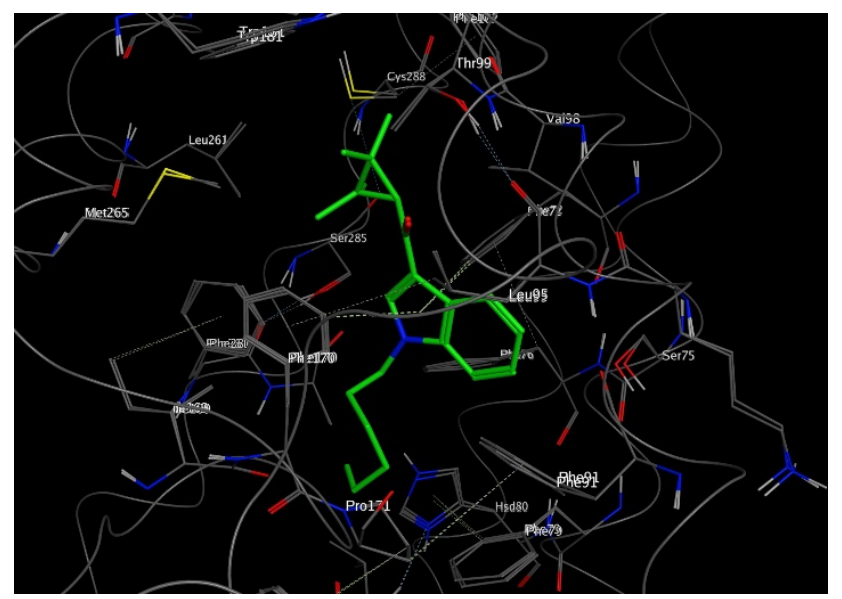

Figure 3. Binding pose of XLR-11 in CB1 active conformation.

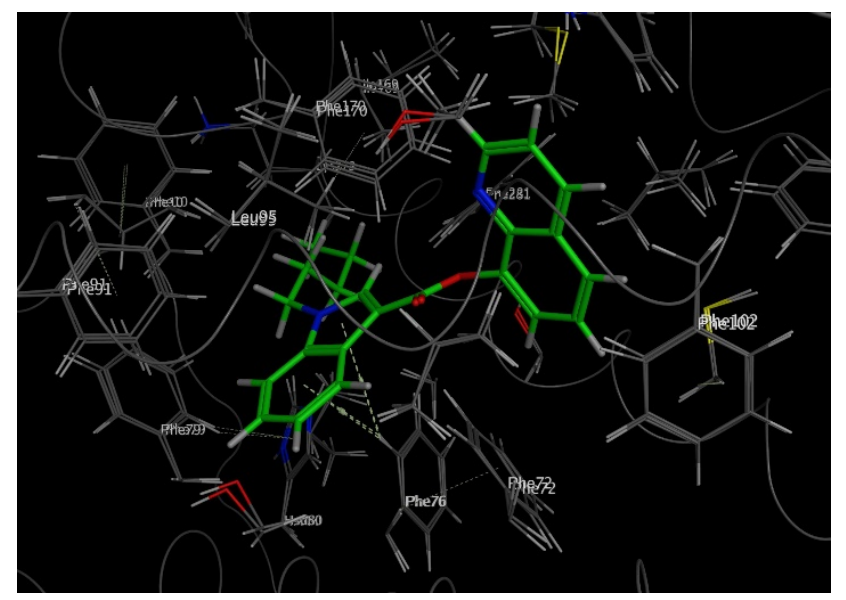

Figure 4. Binding pose of $\mathrm{PB}-22$ in $\mathrm{CB} 1$ active conformation.

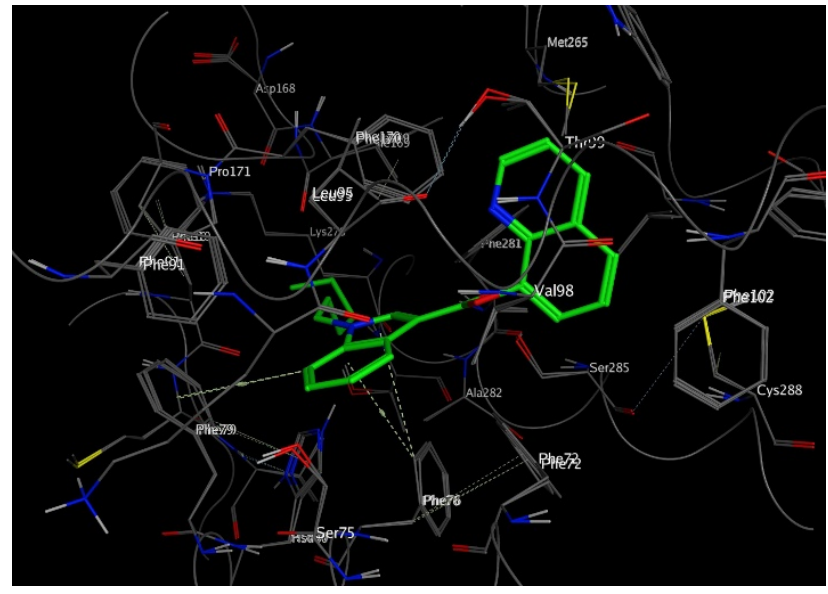

Figure 5. Binding pose of 5F-PB-22 in $\mathrm{CB} 1$ active conformation.

The main interactions between the above ligands and amino acid residues of the CB1 receptor are shown in Figures 6-8. The data obtained show that the main amino acid residues forming the binding pocket and causing interactions with the ligands at the orthosteric binding site in the $\mathrm{CB} 1$ active conformation are Phe10, Phe72, Ser75, Phe76, Phe79, Phe91, Leu95, Val98, Thr99, Phe102, Ile169, Phe170, Trp181, Met265, Lys278, Phe281, Ala282, Ser285, Cys288.

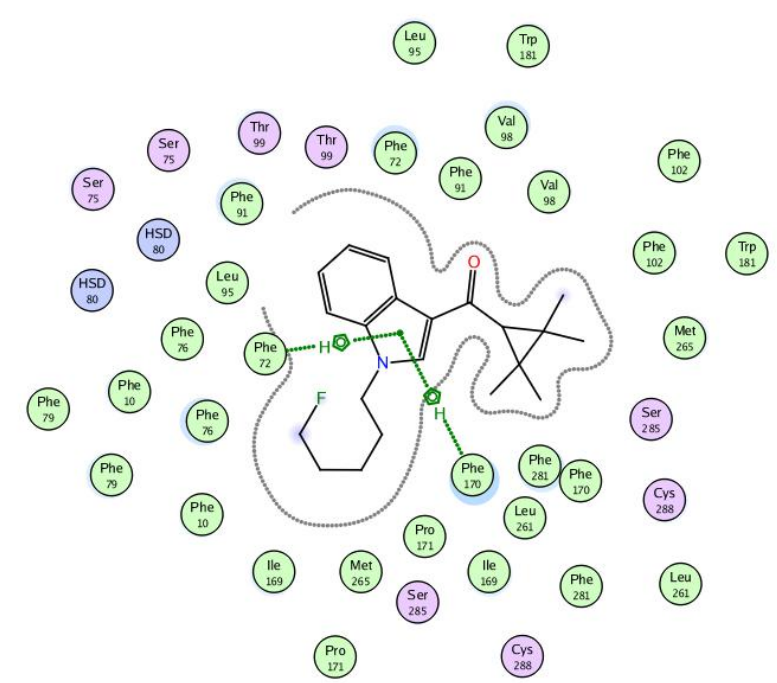

Figure 6. Ligand interactions of XLR-11 in CB1 active conformation. 


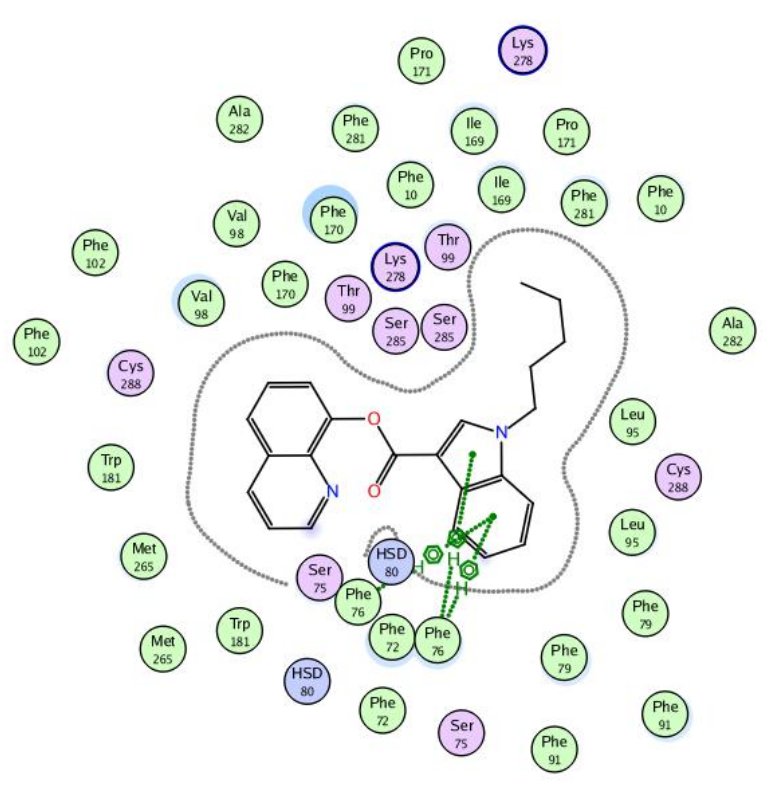

Figure 7. Ligand interactions of $\mathrm{PB}-22$ in $\mathrm{CB} 1$ active conformation.

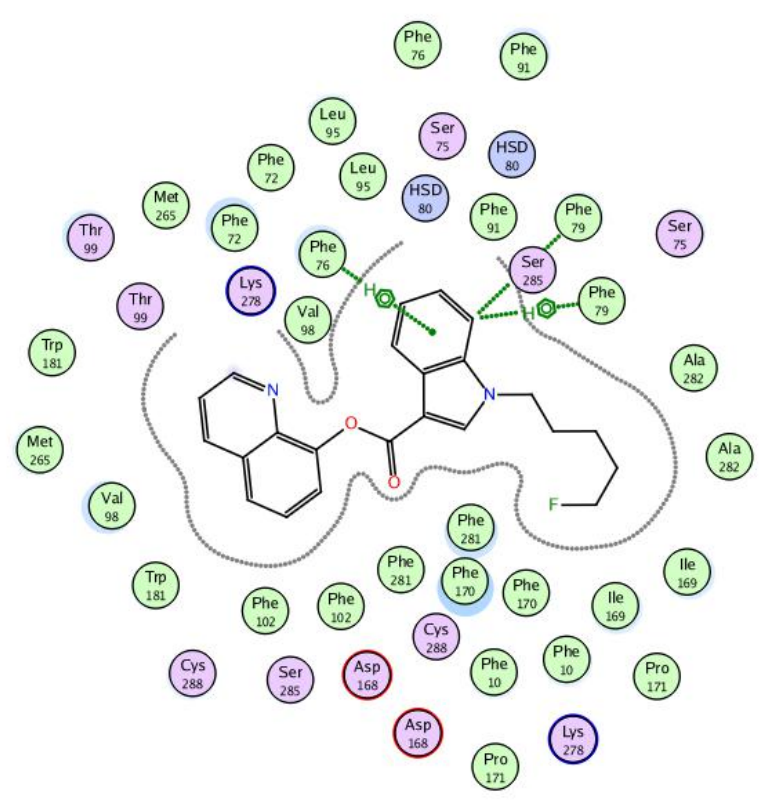

Figure 8. Ligand interactions of 5F-PB-22 in CB1 active conformation.

Thus, the main interactions between the studied agonists and the receptor are hydrophobic and pi-stacking. All considered agonists, except THC, contain $N$-pentylindole fragment in their structures. In this way, the pentyl group and aromatic fragments are mainly responsible for hydrophobic interactions with the receptor, and the indole fragment is responsible for pi-stacking interactions. It should be noted that either the pyrrole core (in XLR-11, Figures 3,6) or the benzene core (in 5F-PB-22, Figures 5,8), or both indole condensed rings simultaneously (in PB-22, Figures 4,7), can participate in stacking interactions.

Sometimes hydrophilic interactions also occur. For example, the ester group in PB-22 is in close proximity to polar amino acid residues such as Thr99, Lys278 and Ser285 (Figures 4,7). Interestingly, for the fluorinated analogue of this agonist, 5F-PB-22, such interactions are already absent (Figures 5,8). This fact can be explained by the different arrangement of the pentyl (in PB-22) and fluoropentyl (in 5F-PB22) tails in the binding pocket, and, as a result, the spatial closure of the 5F-PB-22 ester group.

Next, we carried out the comparative analysis for the studied agonists affinity parameters to $\mathrm{CB} 1$ receptor and for their binding energies calculated via molecular docking. The dissociation constant of the ligand-receptor complex $\left(K_{i}\right)$ and the half maximal effective concentration $\left(\mathrm{EC}_{50}\right)$ were chosen as affinity parameters. High-affinity ligand binding implies that a relatively low concentration of a ligand is adequate to maximally occupy a ligand-binding site and trigger a physiological response. The lower the 
$K_{i}$ is, the more likely there will be a chemical reaction between the ligand and the receptor. Low-affinity binding (high $K_{i}$ values) implies that a relatively high concentration of a ligand is required before the binding site is maximally occupied and the maximum physiological response to the ligand is achieved. $\mathrm{EC}_{50}$ refers to the concentration of ligand, which induces a response halfway between the baseline and maximum after a specified exposure time. It is commonly used as a measure of a drug's potency. A comparison of these two parameters with the calculated binding energies of agonists with the CB1 receptor (S-score) is given in the Table 1.

Table 1. Comparison of CB1 receptor affinity parameters and binding energy from docking for the agonists studied

\begin{tabular}{|c|c|c|c|}
\hline Agonist & $\begin{array}{c}K_{i} \\
(n M)\end{array}$ & $\begin{array}{l}\mathrm{EC}_{50} \\
(n M)\end{array}$ & $\begin{array}{l}\text { S-score } \\
\text { (kcal/mol) }\end{array}$ \\
\hline THC & 13.7 & 250 & -9.29 \\
\hline JWH-018 & 9.5 & 102 & -9.05 \\
\hline AM-2201 & 1.0 & 38 & -9.25 \\
\hline UR-144 & 29 & 421 & -9.19 \\
\hline XLR-11 & 24 & 98 & -9.28 \\
\hline PB-22 & 1.5 & 5.1 & -9.41 \\
\hline 5F-PB-22 & 0.5 & 2.8 & -9.45 \\
\hline
\end{tabular}

The following conclusion can be drawn from the obtained results: there is no strict correlation between the actual binding parameters and the binding energy estimated as docking S score. However, the highest affinity agonists (PB-22 and 5F-PB-22) also have the highest S-scores. In addition, for all $\mathrm{N}$ pentylindole derivatives studied, one regularity is observed: for monofluorinated analogues, the S-score is always slightly higher than for fluorine-free ligands. Interestingly, this clearly coincides with the correlation of affinity parameters for the corresponding fluorinefree/monofluorinated agonist pairs.

In addition, it is easy to see that the S-score values for all the $N$-pentylindole derivatives studied are in the range of $-9.29 \pm 0.25 \mathrm{kcal} / \mathrm{mol}$, where the first value corresponds to the S-score for THC. This result is consistent with the fact that all of these substances, like THC, are proven agonists of the cannabinoid receptor $\mathrm{CB} 1$.

\section{Molecular dynamics results}

Using MBAR method, as implemented in gmx bar utility, free energies of decoupling a ligand from protein, $\Delta G$ ecouple protein, and from water, $\Delta G$ ecouple water, were computed. Binding free energy denoted $\Delta G_{\text {bind }}$ (BAR) was computed as the difference between the stated values (Eq. 1). The results for both forcefields are collected in Table 2. Due to the computational cost of simulations, for CHARMM36 force field, only two ligands (THC and UR-144) were examined.

$\Delta G_{\text {bind }}(\mathrm{BAR})=\Delta G_{\text {decouple water }}-\Delta G_{\text {decouple protein }}(1)$ 
Table 2. Comparison of CB1 receptor affinity parameters and MBAR binding energy estimate for the agonists

\begin{tabular}{|l|l|l|}
\hline \multicolumn{1}{|c|}{ Ligand } & \multicolumn{2}{c|}{$\Delta G_{\text {bind }}$ (BAR), kcal/mol } \\
\hline Forcefield & OPLS-AA/M & CHARMM36 \\
\hline THC & $-22.6 \pm 1.5$ & $-26.6 \pm 1.8$ \\
\hline PB-22 & $-20.3 \pm 1.1$ & \\
\hline 5F-PB-22 & $-15.8 \pm 1.1$ & \\
\hline XLR-11 & $-15.3 \pm 1.4$ & \\
\hline UR-144 & $-15.1 \pm 0.9$ & $-19.1 \pm 0.8$ \\
\hline AM-2201 & $-8.1 \pm 1.6$ & \\
\hline JWH-018 & $-7.6 \pm 1.2$ & \\
\hline
\end{tabular}

The $\Delta G_{b i n d}$ values computed via MMPBSA approach are listed in Table 3.

Table 3. Comparison of CB1 receptor affinity parameters and MMPBSA binding energy estimatie for the agonists

\begin{tabular}{|l|l|l|}
\hline \multicolumn{1}{|c|}{ Ligand } & \multicolumn{2}{c|}{$\Delta G_{\text {bind }}$ (MMPBSA), kcal/mol } \\
\hline \multicolumn{1}{|c|}{ Forcefield } & OPLS-AA/M & CHARMM36 \\
\hline THC & $-42.7 \pm 3.3$ & $-37,3 \pm 2,5$ \\
\hline PB-22 & $-43.7 \pm 2.5$ & $-32.6 \pm 2.7$ \\
\hline 5F-PB-22 & $-40.6 \pm 2.5$ & $-32.6 \pm 2.6$ \\
\hline XLR-11 & $-40.4 \pm 2.7$ & $-33.5 \pm 2.7$ \\
\hline UR-144 & $-40.4 \pm 2.1$ & $-33,1 \pm 2,5$ \\
\hline AM-2201 & $-43.3 \pm 2.8$ & $-33.8 \pm 2.5$ \\
\hline JWH-018 & $-44.5 \pm 2.5$ & $-36.6 \pm 2.6$ \\
\hline
\end{tabular}

Surprisingly, among all the characteristics computed here, only $\Delta G_{\text {bind }}$ (BAR) considerably varies between the ligands, while the others (S-score and $\Delta G_{\text {bind }}$

(MMPBSA)) are almost equal for all of them having the spread within the range of uncertainty. However, no evident correlation is present between $\Delta G_{b i n d}(\mathrm{BAR})$ and experimental affinity parameters of ligands $\left(\mathrm{EC} 50, K_{i}\right)$, as can be seen from comparison (Figure 9). The most striking case is UR-144 that has the highest $\mathrm{EC}_{50}$ and $K$ among the examined set but shows moderate $\Delta G_{b i n d}(\mathrm{BAR})$.

Turning to another forcefield does not improve the correlation. Both the absolute values $\Delta G_{b i n d}$ (MMPBSA) of ligands and their order differ in the two forcefields studied, while the spread is similarly small $(\sim 3 \mathrm{kcal} / \mathrm{mol}$ between the lowest and the highest values) and the uncertainty is of close magnitude $(\sim 2.6$ $\mathrm{kcal} / \mathrm{mol}$ ). The same situation is observed for $\Delta G_{b i n d}(\mathrm{BAR})$, as well.

As a result, as it was shown for molecular docking before, two MD-based methods of computing binding free energy tested here appeared to be able just to determine that all examined compounds show high affinity to $\mathrm{CB} 1$ (i.e. are agonists) but failed to accurately distinguish between more and less active agonists. 


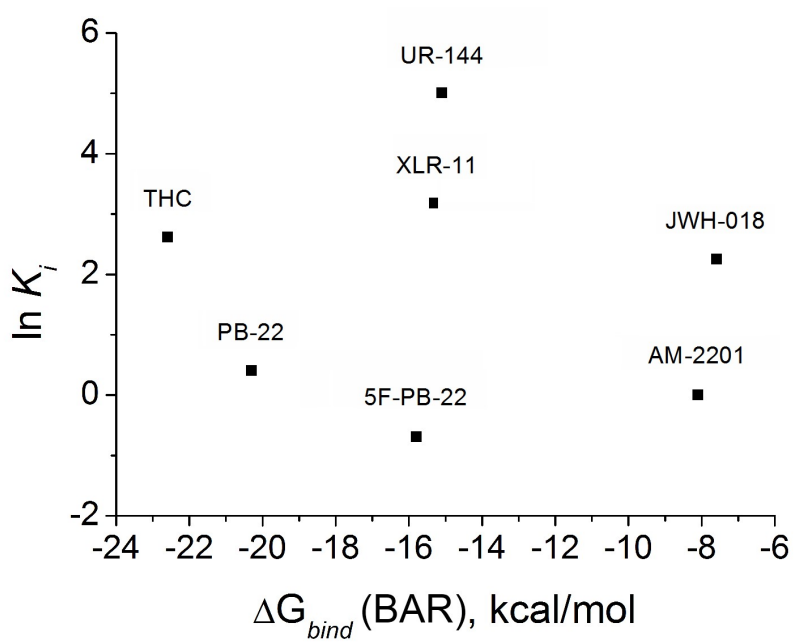

Figure 9. In $K_{i}$ values of the ligands plotted versus their $\Delta \mathrm{G}_{\text {bind }}$ (BAR), computed using, OPLS-AA/M forcefield.

It is difficult to identify the reasons for this issue. Likely, it is not a result of forcefield choice, because two representatives shown similar behavior. The simulation time was several ns that should be enough to sample orientations of a ligand in such constrained space. For $\Delta \mathrm{G}_{\text {bind }}$ (MMPBSA), the uncertainty has to decrease one order of magnitude in order to make the values for various ligands significantly different, and they still will differ slightly by magnitude. Yet, for MBAR, this duration may still be insufficient. Also, a tighter $\lambda$ schedule may be important for accurate estimations. However, fulfilling both options will drastically increase computational cost of the method making it unsuitable for application to large sets of ligands of interest.

A possible source of systematic error is the exclusion of water molecules from the cavity. A presence of a single water molecule may lead to large energy shift in ligand binding energy if formation of a hydrogen bond occurs. Examining this option requires search for a place a water molecule may reside near the ligand. Also, it will not affect the $\Delta \mathrm{G}_{\text {bind }}$ (MMPBSA) values because in these computations, all water is treated implicitly.

\section{Conclusions}

Extensive computational estimation of affinity of a set of agonists to CB1 receptor by three different methods was done. However, no correlation between the calculated binding free energy and experimentally measured affinity parameters $\left(\mathrm{EC}_{50}, K_{i}\right)$ was observed. As a result, the problem of robust discrimination of small organic molecules with respect to affinity to CB1 receptor by means of computational methods remains open. It will be the subject for our following investigations.

\section{Acknowledgements}

Authors thank Ministry of Education and Science of Ukraine for financial support in the frame of project "Molecular docking for express identification of new potential drugs" (0119U002550) and to Dr. Wookyung Yu (Department of Brain Science, Daegu Gyeongbuk Institute of Science and Technology, Daegu, South Korea) for provided files of reconstructed structures of $\mathrm{CB} 1$ protein.

\section{References}

[1] Zou S, Kumar U. Cannabinoid Receptors and the Endocannabinoid System: Signaling and Function in the Central Nervous System. Int. J. Mol. Sci. 2018;19(3):833. 
[2] Yin A, Wang F, Zhang X. Integrating endocannabinoid signaling in the regulation of anxiety and depression. Acta Pharmacologica Sinica. 2019;40:336-341.

[3] Silvestri C, Di Marzo V. The Endocannabinoid System in Energy Homeostasis and the Etiopathology of Metabolic Disorders. Cell Metab. 2013;17(4):475-90. doi: 10.1016/j.cmet.2013.03.001

[4] Casteels C, Rawaha A, Vandenbulcke M, Vandenberghe W, Van Laere K. Cannabinoids and Huntington's disease. In: Fattore L. Cannabinoids in Neurologic and Mental Disease. San Diego: Elsevier Science Publishing Co Inc; 2015. p. 61-97.

[5] Kolb B, Saber H, Fadel H, Rajah G. The endocannabinoid system and stroke: A focused review. Brain Circ. 2019;5(1):1-7. doi:10.4103/bc.bc_29_18

[6] Hourani W, Alexander SPH. Cannabinoid ligands, receptors and enzymes: Pharmacological tools and therapeutic potential. Brain and Neuroscience Advances. 2018;2:1-8. doi: 10.1177/2398212818783908.

[7] Carroll FI, Lewin AH, Mascarella SW, Seltzman HH, Reddy P. Designer drugs: a medicinal chemistry perspective. A. Ann N Y. Acad Sci. 2012;1248:18-38. doi: $10.1111 /$ j.17496632.2011.06199.x.

[8] Wiley JL, Marusich JA, Huffman JW, Balster RL, Thomas BF. Hijacking of Basic Research: The Case of Synthetic Cannabinoids. Methods Rep RTI Press. 2011;2011:17971.doi:10.3768/rtipress.2011.op.0007.1111

[9] Cohen K, Weinstein AM. Synthetic and Nonsynthetic Cannabinoid Drugs and Their Adverse Effects A Review From Public Health Prospective. Front Public Health. 2018;6:162-170. doi:10.3389/fpubh.2018.00162

[10] Weinstein AM, Rosca P, Fattore L, London ED. Synthetic Cathinone and Cannabinoid Designer Drugs Pose a Major Risk for Public Health. Front Psychiatry. 2017;8:156-166. doi:10.3389/fpsyt.2017.00156

[11] Paulke A, Proschak E, Sommer K, Achenbach J, Wunder C, Toennes SW. Synthetic cannabinoids: In silico prediction of the cannabinoid receptor 1 affinity by a quantitative structure-activity relationship model. Toxicol Lett. 2016;245:1-6. doi: 10.1016/j.toxlet.2016.01.001.

[12] Hua T, Vemuri K, Nikas SP, Laprairie RB, Wu Y, Qu L, Pu M, Korde A, Jiang S, Ho JH, Han GW, Ding K, Li X, Liu H, Hanson MA, Zhao S, Bohn LM, Makriyannis A, Stevens RC, Liu ZJ. Crystal structures of agonist-bound human cannabinoid receptor CB1. Nature 2017;547(7664):468-471.

[13] Krishna KK, Shalev-Benami M, Robertson MJ, Hu H, Banister SD, Hollingsworth SA, Latorraca NR, Kato HE, Hilger D, Maeda S, Weis WI, Farrens DL, Dror RO, Malhotra SV, Kobilka BK, Skiniotis G. Structure of a Signaling Cannabinoid Receptor 1-G Protein Complex. Cell. 2019;176(3):448-458. doi: 10.1016/j.cell.2018.11.040

[14] Li X, Hua T, Vemuri K, Ho JH, Wu Y, Wu L, Popov P, Benchama O, Zvonok N, Locke K, Qu L, Han GW, Iyer MR, Cinar R, Coffey NJ, Wang J, Wu M, Katritch V, Zhao S, Kunos G, Bohn LM, Makriyannis A, Stevens RC, Liu ZJ. Crystal Structure of the Human Cannabinoid Receptor CB2, Cell. 2019;176(3):459-467. https://doi.org/10.1016/j.cell.2018.12.011

[15] Hurst DP, Garai S, Kulkarni PM, Schaffer PC, Reggio PH, Thakur GA. Identification of CB1 Receptor Allosteric Sites Using Force-Biased MMC Simulated Annealing and Validation by Structure-Activity Relationship Studies. ACS Med. Chem. Lett. 2019;10(8):1216-1221.

[16] Loo JSE, Murali L, Lee SS, Kueh ALW, Alexander SPH. Ligand discrimination during virtual screening of the $\mathrm{CB} 1$ cannabinoid receptor crystal structures following cross-docking and microsecond molecular dynamics simulations. RSC Adv. 2019;9:15949-15956.

[17] Jung SW, Cho AE, Yu W. Exploring the Ligand Efficacy of Cannabinoid Receptor 1 (CB1) using Molecular Dynamics Simulations. Sci Rep. 2018;8(1):13787-13797. 
[18] Wang C, Greene D, Xiao L, Qi R, Luo R. Recent Developments and Applications of the MMPBSA Method. Front. Mol. Biosci. 2018;4:87.

[19] Shirts MR, Chodera JD. Statistically optimal analysis of samples from multiple equilibrium states. J. Chem. Phys. 2008;129:129105.

[20] Williams-Noonan BJ, Yuriev E, Chalmers DK. Free Energy Methods in Drug Design: Prospects of "Alchemical Perturbation" in Medicinal Chemistry; J. Med. Chem. 2018;61(3):638-649.

[21] Molecular Operating Environment (MOE), 2014.09; Chemical Computing Group Inc., 1010 Sherbooke St. West, Suite \#910, Montreal, QC, Canada, H3A 2R7, 2014.

[22] Naïm M, Bhat S, Rankin KN, Dennis S, Chowdhury SF, Siddiqi I, Drabik P, Sulea T, Bayly CI, Jakalian A, Purisima EO. Solvated interaction energy (SIE) for scoring protein-ligand binding affinities. 1. Exploring the parameter space. J. Chem. Inf. Model. 2007;47(1):122-133.

[23] Huang J, MacKerell AD Jr. CHARMM36 all-atom additive protein force field: validation based on comparison to NMR data. J Comput Chem. 2013;34(25):2135-45.

[24] Vanommeslaeghe K, Hatcher E, Acharya C, et al. CHARMM general force field: A force field for drug-like molecules compatible with the CHARMM allatom additive biological force fields. J Comput Chem. 2010;31(4):671-690.

[25] Robertson MJ, Tirado-Rives J, Jorgensen WL. Improved peptide and protein torsional energetics with the OPLS-AA force field. J. Chem. Theory Comput. 2015;11:3499-3509.

[26] Kulig W, Pasenkiewicz-Gierula M, Róg T. Topologies, structures and parameter files for lipid simulations in GROMACS with the OPLS-aa force field: DPPC, POPC, DOPC, PEPC, and cholesterol. Data in Brief 2015;5:333-336.

[27] Maciejewski A, Pasenkiewicz-Gierula M, Cramariuc O, Vattulainen I, Róg R. Refined OPLS-AA force field for saturated phosphatidylcholine bilayers at full hydration. J. Phys. Chem. B 2014;118:4571-4581.

[28] MD Dodda LS, Cabeza de Vaca I, TiradoRives J, Jorgensen WL. LigParGen web server: an automatic OPLS-AA parameter generator for organic ligands. Nucleic Acids Res. 2017;45(W1):W331-W336.

[29] Kumari R, Kumar R, Open Source Drug Discovery Consortium, Lynn A. g_mmpbsa - A GROMACS tool for high-throughput MM-PBSA calculations. J. Chem. Inf. Model. 2014;54:1951-1962.

[30] Baker NA, Sept D, Joseph S, Holst MJ, McCammon JA. Electrostatics of nanosystems: application to microtubules and the ribosome. Proc. Natl. Acad. Sci. U. S. A. 2001;98(18):10037-10041. 